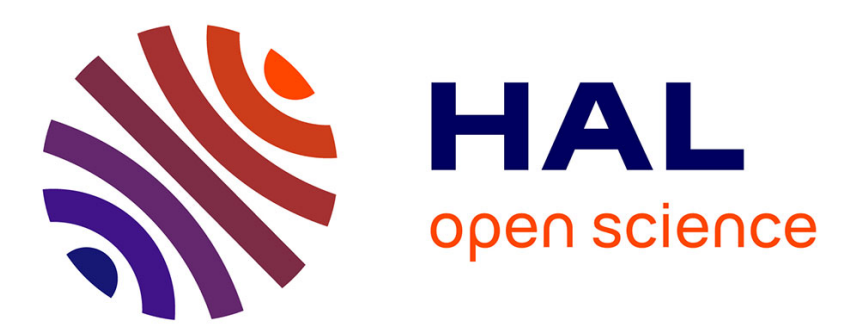

\title{
Relevance of effective bond orders in heterodiatomic molecules and role of the spin-orbit coupling in the AtX $(\mathrm{X}=\mathrm{At}-\mathrm{F})$ series
}

Cecilia Gomez Pech, Pi A.B. Haase, Nicolas Galland, Anastasia Borschevsky, Rémi Maurice

\section{To cite this version:}

Cecilia Gomez Pech, Pi A.B. Haase, Nicolas Galland, Anastasia Borschevsky, Rémi Maurice. Relevance of effective bond orders in heterodiatomic molecules and role of the spin-orbit coupling in the AtX (X=At-F) series. Physical Review A, 2019, 100 (3), pp.032518. 10.1103/PhysRevA.100.032518 . hal-02327747

\section{HAL Id: hal-02327747 \\ https://hal.science/hal-02327747}

Submitted on 19 Mar 2020

HAL is a multi-disciplinary open access archive for the deposit and dissemination of scientific research documents, whether they are published or not. The documents may come from teaching and research institutions in France or abroad, or from public or private research centers.
L'archive ouverte pluridisciplinaire HAL, est destinée au dépôt et à la diffusion de documents scientifiques de niveau recherche, publiés ou non, émanant des établissements d'enseignement et de recherche français ou étrangers, des laboratoires publics ou privés. 


\title{
Relevance of effective bond orders in heterodiatomic molecules and role of the spin-orbit coupling in the $\operatorname{At} X(X=A t-F)$ series
}

\author{
Cecilia Gomez Pech $\odot,{ }^{1,2}$ Pi A. B. Haase $\odot,{ }^{3}$ Nicolas Galland $\odot,{ }^{2}$ Anastasia Borschevsky, ${ }^{3, *}$ and Rémi Maurice $\odot^{1, \dagger}$ \\ ${ }^{1}$ SUBATECH, UMR CNRS 6457, IN2P3/IMT Atlantique/Université de Nantes, 4 rue A. Kastler, 44307 Nantes Cedex 3, France \\ ${ }^{2}$ CEISAM, UMR CNRS 6230, Université de Nantes, 2 rue de la Houssinière, 44322 Nantes Cedex 3, France \\ ${ }^{3}$ Van Swinderen Institute for Particle Physics and Gravity, University of Groningen, Nijenborgh 4, 9747AG Groningen, The Netherlands
}

(Received 10 July 2019; published 30 September 2019)

\begin{abstract}
Scalar and spin-dependent relativistic effects can influence the geometries and wave functions of the ground and excited states of molecular systems in a way that is not always trivial. However, it is still common for researchers, in particular within the quantum chemistry community, to neglect the spin-dependent effects while discussing the binding between atoms in heavy-element systems. Within multiconfigurational self-consistent field frameworks, the binding in diatomic molecules can be derived from the occupation of the natural orbitals, which by definition form a basis that diagonalizes the one-body density matrix. This does not fully prevent arbitrariness, and the first objective of the present paper will be to review the concept of effective bond order, in particular with respect to the rounding up rule. Then, the respective roles of the scalar and the spin-dependent relativistic effects on the bond lengths are investigated by means of state-of-the-art nonrelativistic, scalarrelativistic, and exact two-component coupled-cluster calculations, providing reference molecular geometries for the whole At $X(X=\mathrm{At}-\mathrm{F})$ series. A diagnostic of relevance for defining effective bond orders in heterodiatomic molecules is introduced and applied to this series, showing that the more dissymmetric the system, the less defined the effective bond order is. Finally, the role of the spin-orbit coupling on the effective bond orders is discussed. AtI appears as a key intermediate in the series in terms of the ground-state $\pi$ bonding or antibonding character. Although emphasis will be put on ground states, the present methodology is readily applicable to the description of excited states.
\end{abstract}

DOI: 10.1103/PhysRevA.100.032518

\section{INTRODUCTION}

The description of the binding between atoms is a longstanding issue in quantum chemistry and in molecular physics. Without attempting an exhaustive description of the many philosophies that may be followed, two main strategies emerge: (i) discussing properties of the sole bound system (such as the interatomic distances, atomic charges, an approximate wave function for it, etc.) or (ii) discussing joint properties of both the bound and the dissociated system (the most elementary one being the binding energy, although one may also derive contributions to this energy, for instance). Except trivial indicators such as the interatomic distances, the outcomes of the descriptive approaches always suffer from some degree of arbitrariness, and it is hard to make general recommendations on what is a good description of a bond (or not) and how to universally interpret a descriptor value.

From a wave function calculation, it seems natural to try to interpret the resulting wave function of the quantum state of interest. One may then define the role of physical ingredients such as electron correlation, some particular or all the relativistic effects on the wave function by comparing the outcomes of calculations that do or do not include the physical effect(s) of interest [1]. When several configurations

\footnotetext{
*a.borschevsky@rug.nl

†remi.maurice@ subatech.in2p3.fr
}

may play an important role on the wave function of interest, the multiconfigurational wave function approaches that do optimize both configuration interaction (CI) and molecular orbital (MO) coefficients [2] appear well suited.

As pointed out by Löwdin decades ago [3,4], the multiconfigurational wave functions may appear more "compact" when expressed within the basis of natural orbitals (NOs), which by definition make the one-body density matrix diagonal. This characteristic may be exploited for making the computations more efficient (faster convergence) [3,5] and also for facilitating the interpretation of the wave function, for instance, in terms of the effective bond order (EBO) descriptor [6]. However, despite general acceptance of the advantages of the NOs, they may present some limitations $[7,8]$, in particular for systems with more than two electrons.

Actually, although it is known that the spin-orbit coupling may affect chemical bonds [1], it is quite common in the quantum chemistry community to neglect it when discussing the binding in heavy-element systems [9-11]. Nevertheless, the inclusion of the spin-orbit coupling (SOC) in the computation of EBOs is quite straightforward and has been first reported by Gendron et al. [12] and independently reported soon after by some of us [13]. Furthermore, the EBO concept has also been very recently generalized to the fully relativistic frameworks [14], and thus we consider that it should now be admitted that the SOC must not be neglected while computing EBOs in heavy-element systems. 
Despite these recent efforts, several issues remain concerning the EBO concept: (i) no rule of thumb has been proposed for defining EBOs in heterodiatomic systems and (ii) the EBOs are often misinterpreted because of the rounding up rule that is at play in the quantum chemistry community. This last issue is in our opinion quite critical since it may question conclusions that have been reported in the literature. Therefore, the first objective of the present paper will be to review the EBO concept and to tackle the issue of the heterodiatomic systems by means of a diagnostic of relevance.

We will discuss the $\operatorname{At} X(X=\mathrm{At}-\mathrm{F})$ case studies because (i) these systems display a heavy element, astatine $(Z=85)$, meaning that significant SOC may occur and (ii) some of these systems are among the rare astatine ones of experimental relevance [15-17], in particular AtI that can form halogenbonded adducts with Lewis bases in solution [18]. Prior to this, the role of the scalar relativistic effects and of the spindependent relativistic effects on the molecular geometries will be highlighted by means of state-of-the-art calculations, providing us with reference geometries for performing the EBO study. Finally, the role of the SOC on the chemical bonds of interest will be discussed in terms of EBO, revealing a nontrivial role of the SOC within the series.

\section{THEORY: DEFINITIONS AND GENERAL COMMENTS}

\section{A. Effective bond order concept for a single multiconfigurational state}

In the $\mathrm{H}_{2}$ molecule, the two "delocalized" $\sigma$ and $\sigma^{*}$ MOs belong to two distinct irreducible representations. Thus they are not allowed to mix, meaning that they display pure bonding and antibonding characters, respectively. If one works within this basis, the ground electronic configuration, $\sigma^{2}$, corresponds to a formal bond order of 1 , independent from the retained geometry (near equilibrium or anywhere else on the potential-energy curve). If one allows the ground-state wave function to be expanded on two configurations, namely $\sigma^{2}$ and $\sigma^{* 2}$, the weights associated with both configurations, $\omega_{b}$ and $\omega_{a}$, respectively, will evolve all along the potential-energy curve, becoming equal at dissociation (neutral dissociative state). If one expresses the one-body density matrix for the ground state within the basis of these two $\sigma$ and $\sigma^{*}$ MOs, it is diagonal. Thus these orbitals are referred to as NOs. The occupation numbers of these two orbitals, $\eta_{b}=2 \times \omega_{b}$ and $\eta_{a}=2 \times \omega_{a}$, thus also evolve all along the potential-energy curve, both of them becoming equal to 1 at dissociation. Therefore, one may define an EBO out of these occupation numbers, such that [6]

$$
\mathrm{EBO}=\frac{\eta_{b}-\eta_{a}}{2} .
$$

This bond descriptor has two main advantages: (i) it gradually tends to zero toward dissociation (vide supra) and (ii) at equilibrium, its difference with the formal bond order directly gives the contribution of electron correlation to the EBO. Naturally, this concept can be extended to any homodinuclear system, simply by summing over all the bonding and all the antibonding active NOs [6]:

$$
\mathrm{EBO}=\frac{\sum_{b} \eta_{b}-\sum_{a} \eta_{a}}{2} .
$$

In the seminal paper [6], all the $\sigma, \pi$, etc. contributions to the EBO are summed, thus only total EBOs are reported. For the sake of detailed discussions, it may be wise though to distinguish all these different contributions, principally since two apparently equal total EBOs may hide quite different bonding schemes. In particular, it is quite clear that one effective $\sigma$ bond is not equivalent to one effective $\pi$ bond, which can be attested by a significant energy difference between two states of these two kinds.

The concept of EBO is particularly interesting since it does not force us to define only integer BOs. Unfortunately, Roos et al. recommended that "in naming the multiplicity of a bond one may then use the lowest integer value larger than the EBO" [6], meaning for instance that the bond multiplicity of $\mathrm{H}_{2}$ is back again to 1 all along the potential-energy curve. Another drawback of this rounding up rule emerges when one considers systems with odd numbers of electrons. For instance, if one applies this definition to the $\mathrm{H}_{2}{ }^{+}$prototype, one concludes for a bond multiplicity of 1 , which is clearly incompatible with the number of electrons in the system. Although we are aware that this issue is an interpretation one, and thus that it may be hard to reach a consensus, we advocate for rounding up or down to the closest integer or half-integer value, if rounding off is performed. Also, if one wants to evidence the consequence of a physical or mechanistic effect on a given bond, it is wiser to perform the rounding off after computing the numerical difference between the two EBOs of interest.

The EBO concept has been originally defined for describing the binding in homodiatomic molecules. However, in sufficiently symmetric situations, it can be readily applied to metal-metal complexes [6]. Also, Bolvin and Wagner have evidenced a $\sigma$ half-bond [19] with an even number of electrons case corresponding to an extended system, meaning that EBOs can also be defined in extended systems via the use of the cluster approach and more interestingly that half-integer bond multiplicities are of course not restricted to systems with odd numbers of electrons. Since the definition of EBOs in heterodiatomic molecules is already problematic (vide infra), this issue also transfers to the case of heterobimetallic complexes. In the present work, we will address the definition of EBOs in heterodiatomic molecules, thus also concluding for potential extension of our methodology to heterobimetallic complexes.

\section{B. Multistate cases: The state-average and the "state-specific" natural orbitals}

When one considers multistate cases, one may choose to express each quantum state with its own set of MOs (statespecific approach) or with a common set of MOs, defined to averagely describe the ensemble of quantum states (stateaverage approach). In this latter approach, which is the one that is followed in this work, the state-average NOs (SANOs) for the ensemble of states are obtained by diagonalizing the weighted sum of the one-body density matrices of all the considered states after having democratically optimized the MOs for the ensemble of states. These MOs differ from the state-specific NOs (SS-NOs), obtained by optimizing the MOs for only one quantum state and by diagonalizing its one-body density matrix, and may differ in the general case from the 


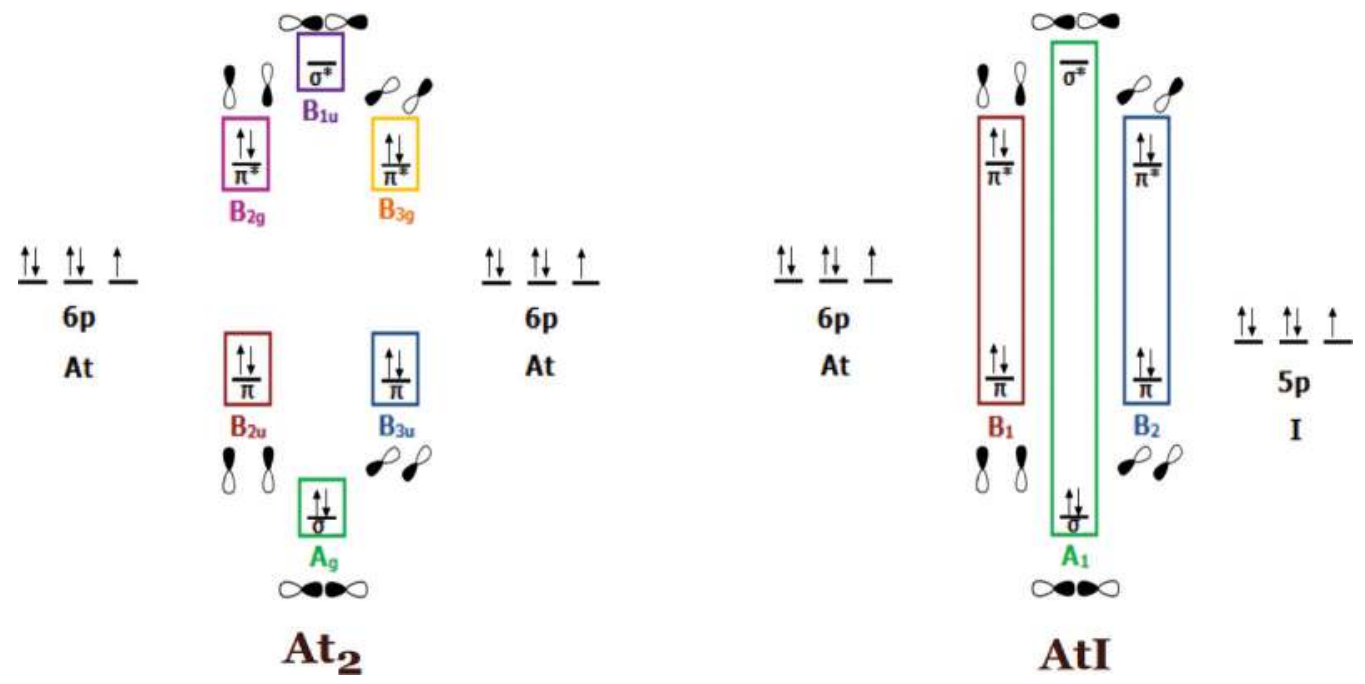

FIG. 1. Partial molecular orbital diagrams of the $\mathrm{At}_{2}$ (left) and AtI (right) molecules. Each color box represents an irreducible representation in the considered symmetry subgroup $\left(D_{2 h}\right.$ for $\mathrm{At}_{2}$ and $C_{2 v}$ for AtI, respectively).

state-average "state-specific" NOs (SA-SS-NOs), obtained from the SA-NOs by diagonalizing the one-body density matrix for the quantum state of interest. However, the SA-SSNOs and the SA-NOs can be strictly identical, for instance, for homodiatomic molecules in the actual $D_{\infty h}$ symmetry group (and in the $D_{2 h}$ symmetry subgroup), providing that orbital mixings are symmetry forbidden. This situation occurs in $\mathrm{At}_{2}$ (see Fig. 1 and Table I for which only the MOs formed from the $6 p$ atomic orbitals are considered), meaning that the previously introduced EBO concept is readily applicable to multistate problems for $\mathrm{At}_{2}$.

In the general case, which already occurs in the AtI system (see Fig. 1 and Table I), orbital mixings are not symmetry forbidden, which has two main consequences for the sake of this work: (i) the SA-SS-NOs and the SA-NOs are not identical anymore and (ii) since orbital mixings between bonding and antibonding orbitals are allowed, the SA-SS-NOs and the SA-NOs are not necessarily fully bonding or fully antibonding, unlike in the homodiatomic situations (this issue also applies to the SS-NOs, i.e., for the description of a single multiconfigurational state). However, a net bonding or antibonding character is assumed for each NO when applying Eq. (2). Defining EBOs in heterodiatomic molecules is therefore a bit ambiguous, although some readily apply Eq. (2), in particular in the case of heterobimetallic complexes [20-22]. In an attempt to solve this issue, we will propose a diagnostic

TABLE I. Irreducible representations associated to the molecular orbitals displayed in Fig. 1 as a function of the considered symmetry group (actual group vs subgroup).

\begin{tabular}{lccccc}
\hline \hline \multirow{2}{*}{ Group } & \multicolumn{2}{c}{$\mathrm{At}_{2}$} & & \multicolumn{2}{c}{$\operatorname{At} X(X \neq \mathrm{At})$} \\
\cline { 2 - 3 } \cline { 5 - 6 }$\sigma_{\infty}$ & $D_{2 h}$ & & $C_{\infty v}$ & $C_{2 v}$ \\
\hline$\sigma^{*}$ & $A_{1 u}$ & $B_{1 u}$ & & $A_{1}$ & $A_{1}$ \\
$\pi$ & $E_{1 g}$ & $B_{2 g} \oplus B_{3 g}$ & & $E_{1}$ & $B_{1} \oplus B_{2}$ \\
$\sigma$ & $E_{1 u}$ & $B_{2 u} \oplus B_{3 u}$ & & $E_{1}$ & $B_{1} \oplus B_{2}$ \\
\hline \hline & $A_{1 g}$ & $A_{g}$ & & $A_{1}$ & $A_{1}$ \\
\hline
\end{tabular}

of relevance for EBOs in heterodiatomic molecules, readily applicable to heterobimetallic complexes.

\section{Relativistic effective bond orders: Spin-orbit coupling a posteriori or a priori}

It is quite straightforward to include scalar relativistic effects while computing EBOs, either via the use of scalarrelativistic pseudopotentials [23] or of a scalar relativistic Hamiltonian [24-31], that must at least differ from the nonrelativistic one on the kinetic-energy terms. The wave function(s) of interest are then expressed in terms of real MOs, and one can directly apply Eq. (2) after having determined the appropriate NOs and the corresponding occupation numbers.

When one wants to include SOC effects, two main routes may be envisaged, by considering (i) that the SOC is a perturbation of the nonrelativistic and scalar-relativistic solutions, and treat it a posteriori [32-34], or (ii) that the SOC is introduced a priori, the wave function of interest being expanded in terms of molecular spinors within a quasirelativistic or a fully relativistic framework.

In the first case, the resulting wave functions are expanded within the basis of real MOs, meaning that the complex character, inherent to relativistic wave functions after the introduction of spin-dependent effects, only appears on the CI coefficients resulting from the spin-orbit configuration interaction (SOCI) step. The SA-NOs may correspond to the SA-SS-NOs of a given spin-orbit quantum state, if it is imposed by symmetry (as in $\mathrm{At}_{2}$ ), or differ, as in the general case (e.g., for the heterodiatomic $\operatorname{At} X$ systems). One can then determine the SA-SS-NOs by diagonalizing the one-body density matrix that is obtained after the introduction of the SOC [35], and afterwards apply Eq. (2) for defining the EBO at the SOCI level [12]. Alternatively, the interpretation of the wave function is possible without explicitly computing the occupation numbers [13], and strictly equivalent to the previous approach providing that the same set of MOs (in practice, the SA-SS-NOs) is used. One can also determine 
EBOs at the uncontracted SOCI level [36], as shown by some of us [13].

In the second case, the complex character of the wave function is introduced within the molecular spinors, which do not display strict $\sigma$, $\pi$, etc. and bonding or antibonding characters, even in homodiatomic molecules. Because of this, Knecht $e t$ al. proposed to define a generalized EBO (gEBO) as follows [14]:

$$
\mathrm{gEBO}=\frac{\sum_{\sigma} \eta_{\sigma}\left(\omega_{b}^{\sigma}-\omega_{a}^{\sigma}\right)}{2},
$$

where the summation runs over the active spinors and where the $\omega_{b}^{\sigma}$ and $\omega_{a}^{\sigma}$ weights give the bonding and antibonding contributions to a given $\sigma$ spinor. Obviously, the gEBO reduces to the previously mentioned EBO for homodiatomic molecules at the scalar relativistic level, since at this level the NOs have pure bonding or antibonding characters. For the $\mathrm{U}_{2}$ molecule, a gEBO of 3.8 was obtained [14], to be compared with the scalar-relativistic EBO of 4.2 of Roos and co-workers $[6,9,37]$. Thus one can conclude that the SOC effectively reduces the bond multiplicity by half a unit in $\mathrm{U}_{2}$, even though both the scalar-relativistic and fully relativistic bond multiplicities may be rounded off to 4 , i.e., one can speak of quadruple bonds in both the cases. Also, since a wrong ground state was initially proposed [9,37], it is clear that quasirelativistic or fully relativistic calculations may be of high importance for determining the correct nature of the ground and excited states in heavy-element systems.

In the present work, exact two-component (X2C) calculations will be performed for computing equilibrium distances, at which the EBOs will be computed at both the scalarrelativistic and SOCI levels. This way, we aim at solving for the issue of heterodiatomic systems in the simpler picture that is obtained while introducing the SOC a posteriori. Note that, in all the studied cases, the nature of the relativistic ground state is similar at both the SOCI and X2C levels, as was also observed in $\mathrm{At}_{2}$ [13]. Therefore, we are confident in the conclusions that we will obtain at this level for the At $X(X=$ At $-\mathrm{F})$ series, and consider the extension of the proposed methodology for heterodiatomic systems to the gEBO framework as a perspective.

\section{COMPUTATIONAL DETAILS}

\section{A. Geometry optimizations}

Equilibrium bond distances have been calculated at the unrestricted coupled cluster level with single, double, and perturbative triple excitations [CCSD(T)] [38] using the DIRAC15 [39] program. The X2C Hamiltonian [30] was employed. The use of this approach was justified by comparison with the four-component level, resulting in negligible changes in bond lengths (less than $0.0025 \AA$ of difference for the tested cases, i.e., $\mathrm{At}_{2}$, AtI, and AtBr). We have also used the corresponding nonrelativistic and scalar-relativistic Hamiltonians in order to highlight the respective roles of the scalar and spin-dependent relativistic effects on the molecular geometries. Dyall's relativistic basis sets of quadruple-zeta quality [40,41], augmented (in an even tempered fashion) with one set of diffuse functions per angular momentum block (AVQZ) were employed. The differences in bond lengths compared to using the nonaugmented VQZ basis sets were in all the test cases less than $0.003 \AA$; thus we consider the results to be sufficiently converged at the AVQZ level for our purposes. All the orbitals within the -20 to 30 a.u. energy range were included in the correlation treatment and the atomic nuclei were modeled by a Gaussian nuclear model [42].

\section{B. Determination of the relativistic and multiconfigurational wave functions}

All the relativistic and multiconfigurational wave functions were determined at the reference $\mathrm{X} 2 \mathrm{C} \operatorname{CCSD}(\mathrm{T})$ geometries. As previously shown [13], the SOCI equilibrium geometries for astatine diatomics are usually in good agreement with the fully relativistic ones, the nature of the ground state being also similar. All the wave function calculations were performed with the MOLPRO program package [43].

The scalar-relativistic wave functions have been obtained by means of state-average complete active space selfconsistent field (SA-CASSCF) calculations [44,45]. Then, scalar-relativistic energies have been computed using the $\mathrm{N}$-electron valence state perturbation theory at second order (NEVPT2) [46-48]. For all the calculations, basis sets of quadruple zeta quality were employed (augcc-pVQZ) [49-52], in conjunction with energy-consistent scalar-relativistic pseudopotentials, supplemented by SOC pseudopotentials for the heaviest atoms, namely $\mathrm{At}, \mathrm{I}$, and $\mathrm{Br}$ [51,52].

In the second step of the calculation, the $\mathbf{H}_{\text {tot }}=\mathbf{E}_{\text {NEVPT2 }}$ $+\mathbf{H}_{\mathrm{SOC}}$ matrix has been diagonalized within the basis of the $M_{S}$ components of the previous scalar-relativistic states (contracted SOCI scheme), generating the perturbed relativistic and multiconfigurational wave functions (the perturbation being here the SOC and this perturbation being variationally treated). Note that it has become standard to dress the diagonal of the $\mathbf{H}_{\mathrm{tot}}=\mathbf{E}_{\mathrm{el}}+\mathbf{H}_{\mathrm{SOC}}$ matrix with more correlated energies than the SA-CASSCF ones, while computing the off-diagonal matrix elements still with the SA-CASSCF wave functions [53,54]. The considered scalar-relativistic states being here well separated in energy, this approximation appears quite justified. Note that the expressions for computing the SOC matrix elements while using pseudopotentials are given elsewhere [23].

In each case, the active space of the SA-CASSCF step comprises 10 electrons within six active orbitals (principally emerging from the $n p$ valence AOs). With one spin-singlet and four spin-triplet scalar-relativistic states, test calculations have shown that more than $99 \%$ of the wave function was recovered as compared to the full set of scalar-relativistic states that can be defined for this active space (21 spin-singlet and 15 spintriplet scalar-relativistic states). Thus the reported results are based on one spin-singlet and four spin-triplet state-averaging space and the following SOCI calculations have retained the spin-singlet and the $4 \times 3$ spin components of the spin-triplet states. Note that in the $\mathrm{At}_{2}$ diatomic molecule, only two scalar-relativistic states are at play, because of symmetry (the lowest-energy spin-triplet state being doubly degenerate and of ungerade symmetry, its components cannot couple to the ground spin-singlet scalar-relativistic state of gerade symmetry via the SOC operator). 
TABLE II. Nonrelativistic, scalar-relativistic, and X2C bond distances $(\AA)$ for the At $X(X=\mathrm{At}-\mathrm{F})$ series obtained with coupled-cluster calculations including single, double, and perturbative triple excitations, $\operatorname{CCSD}(\mathrm{T})$ (this work).

\begin{tabular}{|c|c|c|c|c|c|c|}
\hline \multirow[b]{2}{*}{$X$} & \multirow{2}{*}{$\begin{array}{c}\text { Nonrelativistic } \\
\operatorname{CCSD}(\mathrm{T})\end{array}$} & \multicolumn{3}{|c|}{ Scalar relativistic } & \multirow{2}{*}{$\begin{array}{c}\text { Quasirelativistic } \\
\text { DFT [61] }\end{array}$} & \multirow{2}{*}{$\begin{array}{c}\mathrm{X} 2 \mathrm{C} \\
\mathrm{CCSD}(\mathrm{T})\end{array}$} \\
\hline & & DFT [61] & $\operatorname{CCSD}(\mathrm{T})$ & $\mathrm{CCSD}(\mathrm{T})-\mathrm{F} 12$ [60] & & \\
\hline At & 2.877 & 2.881 & 2.832 & 2.858 & 3.048 & 2.967 \\
\hline I & 2.773 & 2.794 & 2.750 & 2.770 & 2.879 & 2.816 \\
\hline $\mathrm{Br}$ & 2.567 & 2.596 & 2.553 & 2.570 & 2.671 & 2.614 \\
\hline $\mathrm{Cl}$ & 2.416 & 2.449 & 2.411 & 2.420 & 2.524 & 2.471 \\
\hline $\mathrm{F}$ & 1.984 & 2.028 & 1.996 & 2.010 & 2.086 & 2.045 \\
\hline
\end{tabular}

Finally, apart from the $\mathrm{At}_{2}$ case where the SA-NOs are NOs of all the scalar-relativistic and of all the perturbed relativistic and multiconfigurational states, one should stress that the SA-SS-NOs of a given state at the SOCI level are not readily available. The ground-state SA-SS-NOs at the SOCI level were determined by diagonalizing the weighted sum of the density matrices for the five scalar-relativistic states computed at the SA-CASSCF level (the weights corresponding to the sum of the weights associated with the spin components of each scalar-relativistic state in the SOCI wave function).

\section{RESULTS AND DISCUSSION}

\section{A. Role of the scalar-relativistic and spin-dependent effects on the geometries}

The nonrelativistic, scalar-relativistic, and X2C CCSD(T) bond distances for all the $\operatorname{At} X(X=\mathrm{At}-\mathrm{F})$ systems are displayed in Table II. The present values may be compared with previous estimates for these quantities from the literature, for $\mathrm{At}_{2}[13,55-59]$ or for the entire series [60,61]. In the $\mathrm{At}_{2}$ case, the $\mathrm{X} 2 \mathrm{C} \mathrm{CCSD(T)}$ value is notably in good agreement with the contracted and uncontracted SOCI ones, 2.957 and $2.971 \AA$, respectively [13], and the X2C CCSD(T) value of Höfener et al., $3.006 \AA$ [57]. For the rest of the series, only DFT estimates [61] are available for comparison with the new $\mathrm{X} 2 \mathrm{C}$ values. At the scalar-relativistic level, a good agreement is found for the entire series with the very accurate CCSD(T)F12 values proposed by $\mathrm{Hill}$ and $\mathrm{Hu}$ [60], with a mean absolute deviation of $0.017 \AA$, as compared to the deviation of $0.024 \AA$ with DFT. Note that in both cases, i.e., $\operatorname{CCSD}(\mathrm{T})$ or DFT, the deviation from the $\operatorname{CCSD}(\mathrm{T})-\mathrm{F} 12$ values is of the same order of magnitude, while the mean signed deviations display opposite signs. Overall, we thus consider that the new results reported in Table II clearly represent the most accurate and consistent set of bond distances for the entire At $X(X=$ At $-\mathrm{F})$ series, with an appropriate treatment of both relativity and electron correlation.

After having settled the good accuracy of the results reported in Table II, it is worth commenting on the role of the scalar-relativistic and of the spin-dependent (mainly the SOC) relativistic effects on the bond distances of interest. It is clear that in most of the systems $(X=\mathrm{At}-\mathrm{Cl})$, the scalar-relativistic effects lead to a (moderate) bond shortening, as expected for diatomic molecules made of $p$ elements [62]. The AtF molecule appears as an outlier within the series, displaying an apparent bond lengthening induced by scalar-relativistic effects. Actually, this bond distance enlargement is not seen at the Hartree-Fock level, and thus results from the addition and interplay between electron correlation and scalar-relativistic effects. Consequently, it is of different origin than the "classical" scalar-relativistic bond lengthenings that may occur for instance in diatomic molecules with $f$ elements [62]. The differences between the groundstate energies obtained at both the nonrelativistic and scalarrelativistic geometries and at the nonrelativistic and scalarrelativistic Hartree-Fock and $\operatorname{CCSD}(\mathrm{T})$ levels being small (the four resulting energy differences being of the order of $1 \mathrm{~kJ} \mathrm{~mol}^{-1}$ ), it is difficult to draw conclusive insight into the bond lengthening in $\mathrm{AtF}$ and we thus do not discuss it here in further detail.

The role of the SOC on the bond distances is quite systematic in the series, all bonds being significantly lengthened (see Table II). This bond lengthening can be interpreted in a similar way as the role of electron correlation on the bond distance, provided that one considers real MOs for reasoning. As can be seen in Fig. 1, the ground scalar-relativistic configuration for all the systems is $\sigma^{2} \pi^{4} \pi^{* 4}$. The dominant SOC excitations are typically single-excited ones (the two-body SOC terms being usually much smaller than the one-body ones). In these systems, while electron correlation triggers the $\sigma^{2}$ to $\sigma^{* 2}$ double excitation, the SOC leads to the promotion of one electron from the $\pi$ (all the series, as in $\mathrm{At}_{2}$ [13]) or from the $\pi^{*}$ (At $X$ with $X \neq \mathrm{At}$ ) MOs to the $\sigma^{*}$ one. In both cases, the stabilizing effect of the triggered excitations on the total energy of the bound system is favored by diminishing the energy difference between the involved levels, i.e., by lengthening the bond, which is of course in competition with the scalar-relativistic and uncorrelated energy destabilization that arises by moving out of the reference equilibrium geometry. Therefore, it is not surprising to observe a general SOC bond lengthening in the At $X$ series, as for electron correlation in $\mathrm{H}_{2}[63,64]$.

\section{B. Relevance of effective bond orders in heterodiatomic molecules}

The EBOs obtained with the SA-SS-NOs at the SOCI level are given in Table III. Prior to discussing the diagnostic of relevance, it is important to discuss the two contributions to $\mathrm{EBO}_{\text {tot }}$ that arise in the studied cases, i.e., $\mathrm{EBO}_{\sigma}$ and $\mathrm{EBO}_{\pi}$. While one may consider at first sight that $\mathrm{EBO}_{\sigma}$ is practically constant at the SOCI level all along the series (with only minor variations for $\mathrm{At}_{2}$ and $\left.\mathrm{AtF}\right), \mathrm{EBO}_{\pi}$ significantly evolves from a noticeable negative value for $\mathrm{At}_{2}$ ( $\pi$ antibonding character, 
TABLE III. Partial and total effective bond orders for the AtX $(X=$ At $-\mathrm{F})$ series obtained with the SA-SS-NOs at the SOCI level and relative difference of the total bond effective order with the alternative one (see text).

\begin{tabular}{|c|c|c|c|c|c|c|}
\hline \multirow[b]{2}{*}{$X$} & \multirow[b]{2}{*}{$\mathrm{EBO}_{\sigma}$} & \multirow[b]{2}{*}{$\mathrm{EBO}_{\pi}$} & \multirow[b]{2}{*}{$\mathrm{EBO}_{\text {tot }}$} & \multirow[b]{2}{*}{$\mathrm{EBO}_{\text {alt }}$} & \multirow{2}{*}{$\frac{\left|\mathrm{EBO}_{\mathrm{tot}}-\mathrm{EBO}_{\mathrm{alt}}\right|}{\mathrm{EBO}_{\mathrm{tot}}}$} & \multirow[b]{2}{*}{ Relevance } \\
\hline & & & & & & \\
\hline $\mathrm{At}$ & 0.83 & -0.05 & 0.78 & 0.78 & 0 & Full \\
\hline I & 0.87 & 0.00 & 0.87 & 0.85 & $2.3 \%$ & Strong \\
\hline $\mathrm{Br}$ & 0.87 & 0.03 & 0.90 & 0.83 & $7.8 \%$ & Moderate \\
\hline $\mathrm{Cl}$ & 0.87 & 0.06 & 0.93 & 0.79 & $14.1 \%$ & Weak \\
\hline $\mathrm{F}$ & 0.85 & 0.07 & 0.92 & 0.82 & $10.9 \%$ & Weak \\
\hline
\end{tabular}

which was already pointed out in [13]) to a noticeable positive one for AtF. Although this may seem a bit counterintuitive to devotees of the scalar-relativistic picture, this behavior will be elucidated in Sec. IV C. Consequently, $\mathrm{EBO}_{\text {tot }}$ first increases in the series (from $X=$ At to $X=\mathrm{Cl}$ ), before reaching an apparent plateau (for $X=\mathrm{Cl}$ and $X=\mathrm{F}$ ). Again, this behavior was not easily predictable, especially if one neglects the SOC in the reasoning.

In the $\mathrm{At}_{2}$ case, the SA-NOs and the SA-SS-NOs of any of the scalar-relativistic or SOCI solutions perfectly coincide, and each NO has a perfect bonding or antibonding character. As a consequence, if one defines an "alternative" EBO, such as maximizing the bonding and the antibonding characters of the active MOs (see the discussion below), $\mathrm{EBO}_{\text {alt }}$ would be strictly equal to $\mathrm{EBO}_{\text {tot }}$. No deviation between the alternative and the reference value would be observed, and $\mathrm{EBO}_{\text {tot }}$ is thus perfectly relevant. Due to the symmetry lowering with the At $X(X \neq \mathrm{At})$ systems, it cannot be the case and we propose to use the deviation between $\mathrm{EBO}_{\text {alt }}$ and $\mathrm{EBO}_{\text {tot }}$ as a measure of the ambiguity that occurs while defining EBOs in heterodiatomic compounds.

For doing this, we must define how to generate the most bonding and most antibonding active MOs by an appropriate transformation of a given set of active MOs, i.e., how to "rotate" the active MOs and which criteria to use for maximizing the bonding and antibonding characters of each pair of active MOs. As can be seen in Fig. 1 and Table I, in the $C_{2 v}$ symmetry subgroup, the bonding and antibonding orbitals may pairwise mix. For a given pair of active MOs (namely the $\sigma / \sigma^{*}$ pair or one of the two $\pi / \pi^{*}$ ones), a rotation between $\mathrm{MO}_{1}^{0}$ and $\mathrm{MO}_{2}^{0}$ may be defined as follows:

$$
\mathrm{MO}_{1}^{\phi}=\cos (\phi) \mathrm{MO}_{1}^{0}+\sin (\phi) \mathrm{MO}_{2}^{0}
$$

and

$$
\mathrm{MO}_{2}^{\phi}=-\sin (\phi) \mathrm{MO}_{1}^{0}+\cos (\phi) \mathrm{MO}_{2}^{0},
$$

where $\phi$ is the rotation parameter in both equations. For $\phi=\phi^{\max },\left|S_{1}^{i j}\right|$, and $\left|S_{2}^{i j}\right|$, the respective interatomic absolute overlaps between the $I$ and $J$ atoms for the two rotated MOs, defined as

$$
\left|S_{k}^{i j}\right|=\left|\sum_{i} \sum_{j} c_{k}^{i} c_{k}^{j} S^{i j}\right|,
$$

are maximized, one orbital being the most bonding one $\left(S_{k}^{i j}>0\right)$ and the other $\left(S_{k^{\prime}}^{i j}<0\right.$ with $\left.S_{k^{\prime}}^{i j} \neq-S_{k}^{i j}\right)$ the most antibonding one, with $k$ referring to a given rotated MO (still for $\left.\phi=\phi^{\max }\right), c_{k}^{i}$ and $c_{k}^{j}$ being the linear combination of atomic orbital coefficients of orbital $k$ associated with the $i$ and $j$ (contracted) atomic basis functions of atoms $I$ and $J$, respectively, and $S^{i j}$ being the overlap between one atomic basis function of atoms $I$ and one of atom $J$. After quick numerical "scans" over the various possible $\phi$ values (with first a step of $1^{\circ}$, and then refining the maximum value with a step of $0.1^{\circ}$ in appropriate intervals), approximate values of the $\phi^{\max }$ 's have been determined for each $\operatorname{At} X(X \neq \mathrm{At})$ compound and the diagonal elements of the one-body density matrix used to compute $\mathrm{EBO}_{\text {alt }}$. The relative deviations between the $\mathrm{EBO}_{\text {alt }}$ and $\mathrm{EBO}_{\text {tot }}$ values are reported in Table III.

The first important result of the "scans" relates to Lowdin's conjecture [3]: are the SA-SS-NOs actually extremizing the occupation numbers, which would here mean that the absolute values of $\mathrm{EBO}_{\sigma}$ and $\mathrm{EBO}_{\pi}$ are maximized? Since we were unable to find better extrema of the occupation numbers with the previously described scans, we conclude that this is numerically the case, even if we do not strictly have two-electron systems [4,7] (the total $\sigma$ population being $2+\delta$ here with $\delta \approx 0.1 e^{-}$, and the one of each of the two $\pi$ subsystems being $\left.2-\frac{\delta}{2}\right)$. In this sense, the SA-SS-NOs have a well-grounded meaning (since they do maximize the absolute contributions to $\mathrm{EBO}_{\text {tot }}$ ), and so does $\mathrm{EBO}_{\text {tot }}$.

Another important aspect concerns the ambiguity of the definition of EBOs in heterodiatomic compounds. As can be seen in Table III, the deviation between the $\mathrm{EBO}_{\text {alt }}$ and $\mathrm{EBO}_{\text {tot }}$ values increases from $X=$ At to $X=\mathrm{Cl}$, and then decreases from $X=\mathrm{Cl}$ and $X=\mathrm{F}$. Where it comes to interpreting these deviations enters arbitrariness. Some of us have shown that the SOC decreases the EBO in $\mathrm{At}_{2}$ by more than $10 \%$ [13]. Because the ambiguity that affects the EBO must be of smaller magnitude than the physical effects we are interested in, this ambiguity must be kept at least smaller than $10 \%$. Therefore, we qualify the relevance of the $\mathrm{EBO}_{\text {tot }}$ descriptor as "strong" in the case of a deviation between $\mathrm{EBO}_{\text {alt }}$ and $\mathrm{EBO}_{\text {tot }}$ that is comprised between zero and 5\%, "moderate" between 5 and $10 \%$, and "weak" for more than $10 \%$ (see Table III). With this reading grid, the EBO in AtI is strongly meaningful, the one in AtBr moderately meaningful (but still acceptable), and the ones in $\mathrm{AtCl}$ and $\mathrm{AtF}$ are weakly meaningful (though one may still attempt to comment on them). The pointed out situation in $\mathrm{AtCl}$ and $\mathrm{AtF}$ is not surprising owing to the strong asymmetry and the expected significant ionicity of these systems, in contrast to what is expected by assuming the active MOs to have net bonding or antibonding characters (i.e., by thinking of a quite symmetric and "covalent" system).

\section{Role of the spin-orbit coupling on the effective bond orders}

We now continue the analysis of the $\operatorname{At} X(X=$ At F) series by discussing the role of the SOC on the EBOs. The scalar-relativistic EBOs, reported in Table IV, are quite distinct to the SOCI ones. For instance, the $\mathrm{EBO}_{\pi} \mathrm{s}$ are practically zero for all the series, which is not surprising since the ground electronic configuration displays full $\pi$ shells $\left(\sigma^{2} \pi^{4} \pi^{* 4}\right)$. In $\mathrm{At}_{2}$, some of us have already shown that the SOC admixes the ground scalar-relativistic state (a spin-singlet state dominated by the $\sigma^{2} \pi^{4} \pi^{* 4}$ configuration, 
TABLE IV. Partial and total effective bond orders for the At $X$ $(X=$ At $-\mathrm{F})$ series obtained with the SA-SS-NOs at the scalarrelativistic level (SA-CASSCF wave functions). $\triangle \mathrm{EBO}_{\text {tot }}$ is the spinorbit coupling contribution to $\mathrm{EBO}_{\text {tot }}$.

\begin{tabular}{lcccr}
\hline \hline$X$ & $\mathrm{EBO}_{\sigma}$ & $\mathrm{EBO}_{\pi}$ & $\mathrm{EBO}_{\text {tot }}$ & $\Delta \mathrm{EBO}_{\text {tot }}$ \\
\hline $\mathrm{At}$ & 0.90 & 0.00 & 0.90 & -0.12 \\
$\mathrm{I}$ & 0.92 & 0.00 & 0.93 & -0.06 \\
$\mathrm{Br}$ & 0.93 & 0.00 & 0.93 & -0.03 \\
$\mathrm{Cl}$ & 0.93 & 0.00 & 0.94 & -0.01 \\
$\mathrm{~F}$ & 0.92 & 0.00 & 0.92 & 0.00 \\
\hline \hline
\end{tabular}

and of course, to a lesser extent, the $\sigma^{0} \pi^{4} \pi^{* 4} \sigma^{* 2}$ one because of electron correlation) with spin-triplet components of dominant $\sigma^{2} \pi^{3} \pi^{* 4} \sigma^{* 1}$ electronic configuration [13]. These spin-triplet components belong to the highest-energy doubly degenerate scalar-relativistic spin-triplet state that has been computed, the lower-energy one being uncoupled by symmetry to the ground scalar-relativistic state via the SOC operator.

In the $\operatorname{At} X(X \neq \mathrm{At})$ systems, we recall that the SOC can couple the spin components of the two computed doubly degenerate scalar-relativistic spin-triplet states to the ground scalar-relativistic one. While the highest-energy doubly degenerate scalar-relativistic spin-triplet state has the same nature as the spin-triplet state previously discussed for $\mathrm{At}_{2}$ (it essentially corresponds to the $\sigma^{2} \pi^{3} \pi^{* 4} \sigma^{* 1}$ electronic configuration), the nature of the lowest-energy doubly degenerate scalar-relativistic spin-triplet state must be specified: it is dominated by the $\sigma^{2} \pi^{4} \pi^{* 3} \sigma^{* 1}$ electronic configuration, which gives positive contributions to $\mathrm{EBO}_{\pi}$, in contrast to the negative contributions associated with the previous spintriplet state. The more asymmetric the system, the more important the positive contribution is, eventually leading to a change in the $\pi$ character of the ground SOCI state, from $\pi$ antibonding for $X=$ At to $\pi$ bonding for $X=\mathrm{Br}-\mathrm{F}$. Note that this contribution not only depends on the dissymmetry of the system (which translates into the SOC magnitude between the ground scalar-relativistic state and the components of the first doubly degenerate spin-triplet one), but also on the scalar-relativistic excitation energies that may also vary within the series. Since two competing contributions are at play ( $\pi$ bonding and $\pi$ antibonding, respectively) and since the SOC magnitudes and the relevant excitation energies vary in the series, the evolution of $\mathrm{EBO}_{\pi}$ to positive values could not be anticipated without performing the present wave function theory study.

AtI appears as a key intermediate within the series since both competing contributions to $\mathrm{EBO}_{\pi}$ practically cancel out, leading to a nonbonding $\pi$ character for the ground state. However, this does not mean that the role of the SOC on the bonding is dumb, since both competing terms are associated with the promotion of partial electrons from the $\pi$ system to the $\sigma$ one, leading to a significant reduction of $\mathrm{EBO}_{\sigma}$, and thus to an effective "weakening" of the bond.

Intriguingly, the SOC practically leaves $\mathrm{EBO}_{\text {tot }}$ unchanged in $\mathrm{AtCl}$ and $\mathrm{AtF}$, which is not an artifact of the choice of the MOs to compute the EBOs (a similar pattern would be observed with the $\mathrm{EBO}_{\mathrm{alt}} \mathrm{s}$ ). This is actually hiding a strong difference between the two quantities. At the scalarrelativistic level, only $\mathrm{EBO}_{\sigma}$ contributes to $\mathrm{EBO}_{\text {tot }}$, while both $\mathrm{EBO}_{\sigma}$ and $\mathrm{EBO}_{\pi}$ count and sum up to give a similar $\mathrm{EBO}_{\text {tot }}$ at the SOCI level. Since the $\sigma$ and $\pi$ bondings are not equivalent (usually, one would consider that the $\sigma$ bonding is "stronger" than the $\pi$ one), the bonding pictures are then truly distinct at the two considered relativistic levels (scalar relativistic vs SOCI) even though the $\mathrm{EBO}_{\mathrm{tot}} \mathrm{s}$ are similar.

\section{CONCLUDING REMARKS}

The description of the binding between atoms in the ground and excited states of molecules and ions is of everlasting interest in the quantum chemistry and molecular physics communities. In this work, we have contributed to this field by (i) tackling the issue of the definition of EBOs in heterodiatomic molecules, (ii) showing that the SOCI method and the EBO concept can be useful for highlighting in an intelligible way the significant role of the SOC on the binding in heterodiatomic molecules containing a heavy atom such as At $(Z=85)$, (iii) providing reference geometries for a few astatine systems of experimental relevance, and (iv) revealing the role of the scalar and spin-dependent relativistic effects at the molecular geometries of interest. Somehow, this work may be seen as a step toward the nonambiguous definition of gEBOs in heavy heterodiatomic molecules. The reported results may be of broad interest since the issue of defining EBOs in heterodiatomic compounds concerns the entire Periodic Table and because the proposed diagnostic of relevance is also applicable to more compelling systems, such as heterobimetallic complexes (see for instance [20-22]). Finally, we would like to recall that, if an EBO has to be rounded off, we recommend to round up or down to the closest integer or half-integer value, and not to the first superior integer.

\section{ACKNOWLEDGMENTS}

P.A.B.H. and A.B. would like to thank the Center for Information Technology of the University of Groningen for providing access to the Peregrine high performance computing cluster and for their technical support. P.A.B.H. acknowledges funding from the Netherlands Organisation for Scientific Research (NWO) (Grant No. EEDM-166).
[1] K. G. Dyall and K. Faegri, Jr., Introduction to Relativistic Quantum Chemistry (Oxford University Press, New York, 2007).

[2] J. Hinze, J. Chem. Phys. 59, 6424 (1973).

[3] P.-O. Löwdin, Phys. Rev. 97, 1474 (1955).

[4] P.-O. Löwdin and H. Shull, Phys. Rev. 101, 1730 (1956).

[5] E. R. Davidson, Rev. Mod. Phys. 44, 451 (1972).
[6] B. O. Roos, A. C. Borin, and L. Gagliardi, Angew. Chem. Int. Ed. 46, 1469 (2007).

[7] K. Giesbertz, Chem. Phys. Lett. 591, 220 (2014).

[8] N. Suaud and J.-P. Malrieu, Mol. Phys. 115, 2684 (2017).

[9] L. Gagliardi and B. O. Roos, Nature (London) 433, 848 (2005).

[10] A. P. Sattelberger and M. J. A. Johnson, Science 337, 652 (2012). 
[11] X. Li, J. Comput. Chem. 35, 923 (2014).

[12] F. Gendron, B. Le Guennic, and J. Autschbach, Inorg. Chem. 53, 13174 (2014).

[13] R. Maurice, F. Réal, A. S. P. Gomes, V. Vallet, G. Montavon, and N. Galland, J. Chem. Phys. 142, 094305 (2015).

[14] S. Knecht, H. J. A. Jensen, and T. Saue, Nat. Chem. 11, 40 (2019).

[15] E. H. Appelman, E. N. Sloth, and M. H. Studier, Inorg. Chem. 5, 766 (1966).

[16] J. Champion, M. Seydou, A. Sabatié-Gogova, E. Renault, G. Montavon, and N. Galland, Phys. Chem. Chem. Phys. 13, 14984 (2011).

[17] N. Guo, D.-C. Sergentu, D. Teze, J. Champion, G. Montavon, N. Galland, and R. Maurice, Angew. Chem. Int. Ed. 55, 15369 (2016).

[18] N. Guo, R. Maurice, D. Teze, J. Graton, J. Champion, G. Montavon, and N. Galland, Nat. Chem. 10, 428 (2018).

[19] H. Bolvin and F. R. Wagner, Inorg. Chem. 51, 7112 (2012).

[20] L. J. Clouston, R. B. Siedschlag, P. A. Rudd, N. Planas, S. Hu, A. D. Miller, L. Gagliardi, and C. C. Lu, J. Am. Chem. Soc. 135, 13142 (2013).

[21] S. J. Tereniak, R. K. Carlson, L. J. Clouston, V. G. Young, E. Bill, R. Maurice, Y.-S. Chen, H. J. Kim, L. Gagliardi, and C. C. Lu, J. Am. Chem. Soc. 136, 1842 (2014).

[22] R. J. Eisenhart, P. A. Rudd, N. Planas, D. W. Boyce, R. K. Carlson, W. B. Tolman, E. Bill, L. Gagliardi, and C. C. Lu, Inorg. Chem. 54, 7579 (2015).

[23] M. Dolg and X. Cao, Chem. Rev. 112, 403 (2012).

[24] C. Chang, M. Pelissier, and P. Durand, Phys. Scr. 34, 394 (1986).

[25] J.-L. Heully, I. Lindgren, E. Lindroth, S. Lundqvist, and A.-M. Martensson-Pendrill, J. Phys. B 19, 2799 (1986).

[26] E. van Lenthe, E. J. Baerends, and J. G. Snijders, J. Chem. Phys. 99, 4597 (1993).

[27] M. Douglas and N. M. Kroll, Ann. Phys. (NY) 82, 89 (1974).

[28] B. A. Hess, Phys. Rev. A 33, 3742 (1986).

[29] G. Jansen and B. A. Hess, Phys. Rev. A 39, 6016 (1989).

[30] M. Iliaš and T. Saue, J. Chem. Phys. 126, 064102 (2007).

[31] W. Liu and D. Peng, J. Chem. Phys. 131, 031104 (2009).

[32] P.-Å. Malmqvist, B. O. Roos, and B. Schimmelpfennig, Chem. Phys. Lett. 357, 230 (2002).

[33] B. O. Roos and P.- $\AA$. Malmqvist, Phys. Chem. Chem. Phys. 6, 2919 (2004).

[34] D. Ganyushin and F. Neese, J. Chem. Phys. 125, 024103 (2006).

[35] F. Gendron, D. Páez-Hernández, F.-P. Notter, B. Pritchard, H. Bolvin, and J. Autschbach, Chem. Eur. J. 20, 7994 (2014).

[36] V. Vallet, L. Maron, C. Teichteil, and J.-P. Flament, J. Chem. Phys. 113, 1391 (2000).

[37] B. O. Roos, P.-Å. Malmqvist, and L. Gagliardi, J. Am. Chem. Soc. 128, 17000 (2006).

[38] L. Visscher, T. J. Lee, and K. G. Dyall, J. Chem. Phys. 105, 8769 (1996).

[39] L. Visscher, H. J. Aa. Jensen, R. Bast, and T. Saue, with contributions from V. Bakken, K. G. Dyall, S. Dubillard, U. Ekström, E. Eliav, T. Enevoldsen, E. Faßhauer, T. Fleig, O. Foss-gaard, A. S. P. Gomes, E. D. Hedegård, T. Helgaker, J. Henriksson, M. Iliaš, Ch. R. Jacob, S. Knecht, S. Komorovský, O. Kullie, J. K. Lærdahl, C. V. Larsen, Y. S. Lee, H. S. Nataraj, M. K. Nayak, P. Norman, G. Olejniczak, J. Olsen, J. M. H. Olsen, Y. C. Park, J. K. Pedersen, M. Pernpointner, R. di Remigio,
K. Ruud, P. Sałek, B. Schimmelpfennig, A. Shee, J. Sikkema, A. J. Thorvaldsen, J. Thyssen, J. van Stralen, S. Villaume, O. Visser, T. Winther, and S. Yamamoto. DIRAC, a relativistic ab initio electronic structure program, release DIRAC15, 2015; see http://www.diracprogram.org.

[40] K. G. Dyall, Theor. Chem. Acc. 115, 441 (2006).

[41] K. G. Dyall, Theor. Chem. Acc. 135, 128 (2016).

[42] L. Visscher and K. G. Dyall, At. Data Nucl. Data Tables 67, 207 (1997).

[43] H. J. Werner, P. J. Knowles, R. Lindh, F. R. Manby, M. Schtz, P. Celani, T. Korona, A. Mitrushenkov, G. Rauhut, T. B. Adler, R. D. Amos, A. Bernhardsson, A. Berning, D. L. Cooper, M. J. O. Deegan, A. J. Dobbyn, F. Eckert, E. Goll, C. Hampel, G. Hetzer, T. Hrenar, G. Knizia, C. Kppl, Y. Liu, A. W. Lloyd, R. A. Mata, A. J. May, S. J. McNicholas, W. Meyer, M. E. Mura, A. Nicklass, P. Palmieri, K. Pflger, R. Pitzer, M. Reiher, U. Schumann, H. Stoll, A. J. Stone, R. Tarroni, T. Thorsteinsson, M. Wang, and A. Wolf, MOLPRO, A package of ab initio programs.

[44] B. O. Roos, P. R. Taylor, and P. E. Siegbahn, Chem. Phys. 48, 157 (1980).

[45] B. O. Roos, The complete active space self-consistent field method and its applications in electronic structure calculations, in Advances in Chemical Physics (John Wiley \& Son Ltd., Chichester, 1987), pp. 399-445.

[46] C. Angeli, R. Cimiraglia, S. Evangelisti, T. Leininger, and J.-P. Malrieu, J. Chem. Phys. 114, 10252 (2001).

[47] C. Angeli, R. Cimiraglia, and J.-P. Malrieu, Chem. Phys. Lett. 350, 297 (2001).

[48] C. Angeli, R. Cimiraglia, and J.-P. Malrieu, J. Chem. Phys. 117, 9138 (2002).

[49] R. A. Kendall, T. H. Dunning, and R. J. Harrison, J. Chem. Phys. 96, 6796 (1992).

[50] D. E. Woon and T. Dunning, J. Chem. Phys. 98, 1358 (1993).

[51] K. A. Peterson, D. Figgen, E. Goll, H. Stoll, and M. Dolg, J. Chem. Phys. 119, 11113 (2003).

[52] K. A. Peterson, B. C. Shepler, D. Figgen, and H. Stoll, J. Phys. Chem. A 110, 13877 (2006).

[53] C. Teichteil, M. Pélissier, and F. Spiegelmann, Chem. Phys. 81, 273 (1983).

[54] R. Llusar, M. Casarrubios, Z. Barandiarán, and L. Seijo, J. Chem. Phys. 105, 5321 (1996).

[55] L. Visscher and K. G. Dyall, J. Chem. Phys. 104, 9040 (1996).

[56] V. Pershina, A. Borschevsky, J. Anton, and T. Jacob, J. Chem. Phys. 132, 194314 (2010).

[57] S. Höfener, R. Ahlrichs, S. Knecht, and L. Visscher, Chem. Phys. Chem. 13, 3952 (2012).

[58] J. Pilmé, E. Renault, T. Ayed, G. Montavon, and N. Galland, J. Chem. Theory Comput. 8, 2985 (2012).

[59] D.-C. Sergentu, G. David, G. Montavon, R. Maurice, and N. Galland, J. Comput. Chem. 37, 1345 (2016).

[60] J. G. Hill and X. Hu, Chem. Eur. J. 19, 3620 (2013).

[61] J. Pilmé, E. Renault, F. Bassal, M. Amaouch, G. Montavon, and N. Galland, J. Chem. Theory Comput. 10, 4830 (2014).

[62] W. Zou, M. Filatov, and D. Cremer, J. Chem. Phys. 142, 214106 (2015).

[63] D. J. DeFrees, B. A. Levi, S. K. Pollack, W. J. Hehre, J. S. Binkley, and J. A. Pople, J. Am. Chem. Soc. 101, 4085 (1979).

[64] D. J. DeFrees, K. Raghavachari, H. B. Schlegel, and J. A. Pople, J. Am. Chem. Soc. 104, 5576 (1982). 